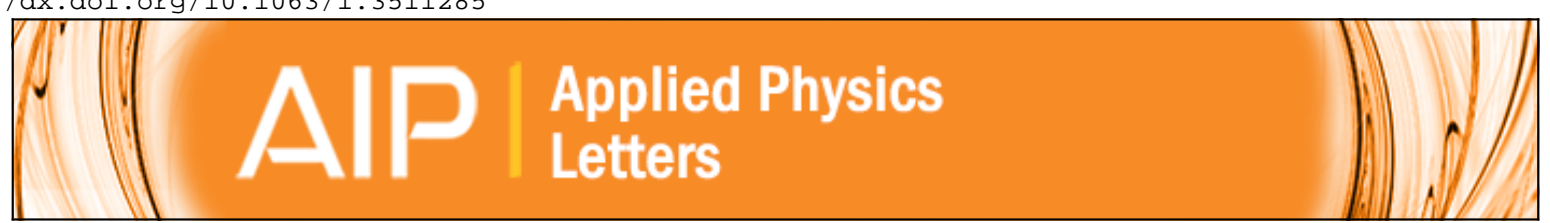

\title{
Effect of intensive and extensive loss factors on the dynamic response of
} magnetoelectric laminates

Kyung-Hoon Cho, Chee-Sung Park, and Shashank Priya

Citation: Applied Physics Letters 97, 182902 (2010); doi: 10.1063/1.3511285

View online: $\mathrm{http} / / / \mathrm{dx}$. doi.org/10.1063/1.3511285

View Table of Contents: http://scitation.aip.org/content/aip/journal/apl/97/18?ver=pdfcov

Published by the AIP Publishing

\section{Over 700 papers \& presentations on} multiphysics simulation 


\title{
Effect of intensive and extensive loss factors on the dynamic response of magnetoelectric laminates
}

\author{
Kyung-Hoon Cho, Chee-Sung Park, and Shashank Priya ${ }^{a)}$ \\ Center for Energy Harvesting Materials and Systems (CEHMS), Materials Science and Engineering, \\ Virginia Tech, Blacksburg, Virginia 24061, USA
}

(Received 12 September 2010; accepted 13 October 2010; published online 3 November 2010)

\begin{abstract}
We report the correlation between intensive and extensive losses in piezoelectric materials with the frequency dependent response of layered magnetoelectric (ME) composites. Three different piezoelectric compositions were synthesized to achieve varying loss characteristics allowing a systematic interpretation of changes in ME coupling in terms of loss components. We clearly demonstrate that intensive dielectric and piezoelectric loss play an important role in controlling the ME sensitivity of layered composites in sub-resonance low frequency range while extensive mechanical loss is dominant factor at resonance condition. Further, the maximum in ME response is obtained at antiresonance frequency of piezoelectrics. (C) 2010 American Institute of Physics. [doi:10.1063/1.3511285]
\end{abstract}

Magnetoelectric (ME) materials provide direct coupling between the magnetic field and electric polarization. ${ }^{1-5}$ In laminate composites, the ME voltage coefficient of longitudinally magnetized and transversely poled system (L-T mode) is given as follows: ${ }^{1}$

$$
\alpha_{E, 31}=\frac{d E_{3}}{d H_{1}}=\frac{n q_{11} g_{31}}{n S_{11}^{E}\left(1-k_{31}^{2}\right)+(1-n) S_{11}^{H}},
$$

where $H_{1}$ is the longitudinally applied magnetic field along 1 axis, $E_{3}$ is the generated electric field perpendicular to $H_{1}$ direction ( 3 axis), $n$ is the volume fraction of the magnetostrictive layer $\left[n=V_{\text {magnetic }} /\left(V_{\text {piezoelectric }}+V_{\text {magnetic }}\right) ; V\right.$ is the volume], $q_{11}$ is the piezomagnetic coefficient, $g_{31}$ is the piezoelectric voltage coefficient, $k_{31}$ is the electromechanical coupling coefficient, and $S_{11}^{E}$ and $S_{11}^{H}$ are the elastic compliances of the piezoelectric and magnetostrictive layers respectively. Since the parameter $g_{31}$ is related to dielectric permittivity by the relation: $g_{31}=k_{31}\left(S_{11}^{E}\right)^{1 / 2}\left(\varepsilon_{33}\right)^{-1 / 2}$ where $\varepsilon_{33}$ is the dielectric permittivity, the magnitude of $\alpha_{E, 31}$ is dependent upon the dielectric behavior.

Intensive losses in piezoelectrics are related to hysteresis when the material is driven forward and backward simultaneously by AC field $(E)$. Generally, this nonlinear behavior can be associated with the influence of mechanical and electrical stresses on domains. The intensive dielectric loss $\left(\tan \delta^{\prime}\right)$ can be simply described by the hysteresis during $180^{\circ}$ polarization reversal under applied electric field, while the mechanical loss $\left(\tan \varphi^{\prime}\right)$ can be related to the hysteresis during $90^{\circ}$ polarization reversal under applied stress. The nonlinearity of dielectric displacement with respect to stress, or strain with respect to electric field, produces harmonic distortion and dissipation and is termed as piezoelectric loss $\left(\tan \theta^{\prime}\right)$. Experimentally, intensive losses can be easily measured from the impedance vs. frequency behavior. The mechanical loss is given by the inverse of mechanical quality factor $\left(\mathrm{Q}_{\mathrm{m}}\right), \tan \delta^{\prime}$ is determined from the phase angle, and $\tan \theta^{\prime}$ can be obtained by following equation:,

${ }^{a)}$ Electronic mail: spriya@vt.edu.

$$
\tan \theta^{\prime}=\frac{\tan \delta^{\prime}+\tan \varphi^{\prime}}{2}+\frac{1}{4}\left(\frac{1}{Q_{r}}-\frac{1}{Q_{a}}\right)\left[1+\left(\frac{1}{k_{31}}-k_{31}\right)^{2} \Omega_{a}^{2}\right]
$$

where $\mathrm{Q}_{\mathrm{r}}\left(=\mathrm{Q}_{\mathrm{m}}\right)$ and $\mathrm{Q}_{\mathrm{a}}$ are mechanical quality factor at resonance $\left(f_{\mathrm{r}}\right)$ and antiresonance $\left(f_{\mathrm{a}}\right)$ frequency, respectively, and $\Omega_{\mathrm{a}}$ is normalized frequency $\Omega_{\mathrm{a}}=\pi f_{\mathrm{a}} l v^{-1}, l$ is length and $v$ is sound velocity. From materials perspective, an interesting question that can be posed is "How do these dynamic loss terms affect the ME coupling in laminates?"

Three different piezoelectric compositions were synthesized for this study: (1) $0.05 \mathrm{~Pb}\left(\mathrm{Mn}_{1 / 3} \mathrm{Sb}_{2 / 3}\right) \mathrm{O}_{3}$ $-0.95 \mathrm{~Pb}\left(\mathrm{Zr}_{0.52} \mathrm{Ti}_{0.48}\right) \mathrm{O}_{3}$ (PMS) representing hard piezoelectrics, (2) 0.4 wt $\% \mathrm{Mn}$ doped $0.2 \mathrm{~Pb}\left(\mathrm{Zn}_{1 / 3} \mathrm{Nb}_{2 / 3}\right) \mathrm{O}_{3}$ $-0.8 \mathrm{~Pb}\left(\mathrm{Zr}_{0.5} \mathrm{Ti}_{0.5}\right) \mathrm{O}_{3}(\mathrm{MPZN})$ as an intermediate composition, and (3) $0.2 \mathrm{~Pb}\left(\mathrm{Zn}_{1 / 3} \mathrm{Nb}_{2 / 3}\right) \mathrm{O}_{3}-0.8 \mathrm{~Pb}\left(\mathrm{Zr}_{0.5} \mathrm{Ti}_{0.5}\right) \mathrm{O}_{3}$ (PZN) representing soft piezoelectrics. Ceramic samples were synthesized by using conventional mixed oxide processing described in detail elsewhere. ${ }^{8,9}$ Electromechanical characterization was conducted by using a precision LCR meter (Agilent 4284A, USA) and impedance analyzer (Agilent 4294A, USA). The measured properties are shown in Table I (sample dimension: $27 \times 5 \times 1 \mathrm{~mm}^{3}$ ). After poling piezoelectric plates, $46 \mu \mathrm{m}$ thick Metglas (2605SA1, MetglasInc., USA) sheet was attached by using epoxy (West System, USA) on top and bottom of the piezoelectric layer. ME measurements were done in L-T mode configuration using lock-in amplifier method.

Figure 1 shows the ME voltage coefficient $\left(\alpha_{E, 31}\right)$ of laminates measured in low $\mathrm{H}_{\mathrm{ac}}$ frequency range of $20 \mathrm{~Hz}$ $\sim 1 \mathrm{kHz}$ under constant $\mathrm{H}_{\mathrm{dc}}(21 \mathrm{Oe})$. All the laminates exhibited maximum $\alpha_{E, 31}$ at the same bias magnetic field of 21

TABLE I. Material parameters of piezoelectric compositions used in this study.

\begin{tabular}{lcccccc}
\hline \hline & $\begin{array}{c}d_{31} \\
(\mathrm{pC} / \mathrm{N})\end{array}$ & $\begin{array}{c}\varepsilon_{33} \\
\left(\times 10^{-9} \mathrm{~F} / \mathrm{m}\right)\end{array}$ & $k_{31}$ & $\mathrm{Q}_{\mathrm{m}}$ & $\begin{array}{c}g_{31} \\
\left(\times 10^{-3} \mathrm{Vm} / \mathrm{N}\right)\end{array}$ & $\begin{array}{c}\mathrm{S}_{11}^{\mathrm{E}} \\
\left(\times 10^{-12} \mathrm{~m}^{2} / \mathrm{N}\right)\end{array}$ \\
\hline PMS & -63 & 5.1 & 0.28 & 2291 & -12.47 & 9.9 \\
MPZN & -121 & 13.8 & 0.28 & 664 & -8.74 & 13 \\
PZN & -204 & 19.3 & 0.37 & 85 & -10.56 & 16 \\
\hline \hline
\end{tabular}




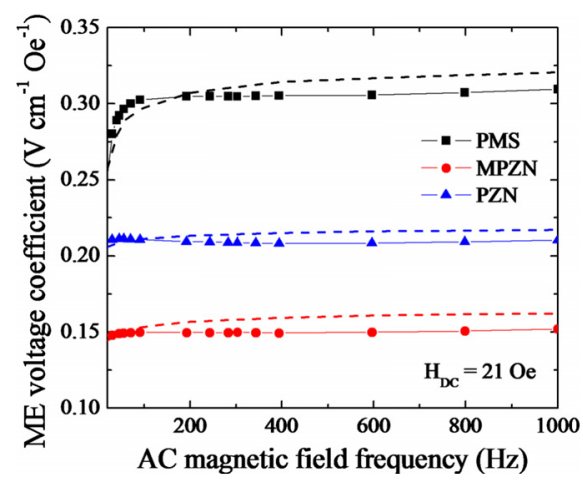

FIG. 1. (Color online) Magnetoelectric voltage coefficient of PMS/Metglas, MPZN/Metglas and PZN/Metglas laminates measured in $\mathrm{H}_{\mathrm{ac}}$ frequency range of $20 \mathrm{~Hz} \sim 1 \mathrm{kHz}$ under $\mathrm{H}_{\mathrm{dc}}$ of $21 \mathrm{Oe}$. Dashed lines indicate calculated magnetoelectric voltage coefficient values by using Eq. (4). It can be clearly seen that the decreasing rate of coefficient of PMS/Metglas is the most rapid in low frequency range.

Oe which is expected as this maxima is related to the peak in piezomagnetic coefficient of Metglas. PMS/Metglas composite exhibited the highest magnitude of $\alpha_{E, 31}$ over the entire frequency range which could be related to its high $g_{31}$ and low $S_{11}^{E}$ (see Table I). Note that $\alpha_{E, 31}$ of PMS/Metglas is more than $0.3 \mathrm{~V} \mathrm{~cm}^{-1} \mathrm{Oe}^{-1}$ although this composite has a very low $n$ value of 0.084 . A rapid drop in $\alpha_{E, 31}$ was observed for PMS/Metglas in the low frequency range below $100 \mathrm{~Hz}$ while MPZN/Metglas showed small drop and PZN/ Metglas showed almost negligible change. The rapid change in ME output voltage in low frequency region represents a serious problem for a magnetic field sensor based on laminates. We demonstrate that the source of this problem is mainly related to the intensive loss factors of piezoelectrics. The consequence of Fig. 1 is that the best piezoelectric composition, solely designed based on real values of electromechanical parameters, may exhibit the worst behavior at low frequencies.

Figure 2 shows the variation in rate of capacitance change $\left(\mathrm{C}-\mathrm{C}_{\mathrm{f}}\right) / \mathrm{C}_{\mathrm{f}}$, where $\mathrm{C}$ is capacitance at a given frequency, and $\mathrm{C}_{\mathrm{f}}$ is capacitance at $1 \mathrm{kHz}$. It can be seen in this figure that at low frequencies the change in loss parameters and relative capacitance change is more significant for PMS composition as compared to MPZN and PZN. Especially below $100 \mathrm{~Hz}, \tan \delta^{\prime}$ and $\tan \theta^{\prime}$ values for PMS were found to increase exponentially with decreasing frequency, while MPZN showed relatively intermediate increase and PZN exhibited negligible change. By comparing the trend of ME response in Fig. 1 and dielectric and piezoelectric loss characteristics in Fig. 2, we propose an empirical variable $g_{31}^{*}$ that

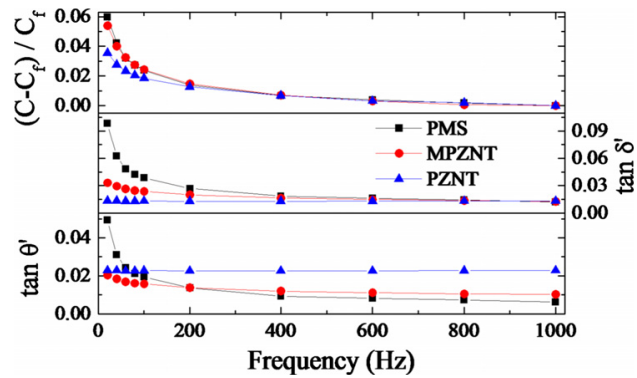

FIG. 2. (Color online) The rate of capacitance change, dielectric loss and piezoelectric loss of PMS, MPZN, and PZN piezoelectric compositions measured in the frequency' range of $20 \mathrm{~Hz} \sim 1 \mathrm{kHz}$.

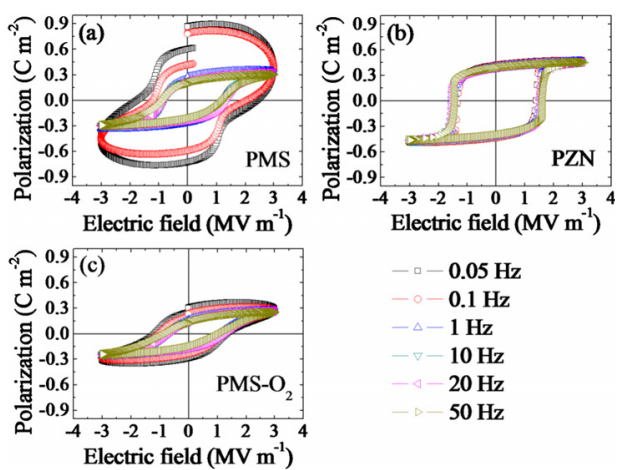

FIG. 3. (Color online) Polarization vs electric field characteristics in low frequency range of (a) PMS sintered in air, (b) PZN sintered in air, and (c) PMS sintered in oxygen atmosphere.

could be substituted for $g_{31}$ in Eq. (1) to derive the ME response as follows:

$$
\begin{aligned}
& g_{31}^{*}=g_{31} \frac{1}{\left(\tan \delta^{\prime}+\tan \theta^{\prime}+\frac{C-C_{f}}{C_{f}}\right)}, \\
& \alpha_{E, 31}=\frac{d E_{3}}{d H_{1}}=\frac{n q_{11} g_{31}^{*}}{n S_{11}^{E}\left(1-k_{31}^{2}\right)+(1-n) S_{11}^{H}} .
\end{aligned}
$$

Dashed lines in Fig. 1 represent the calculated $\alpha_{E, 31}$ by using Eq. (4) which matches very well with the experimental data. Rewriting, Eq. (3) in the form of dielectric constant and expanding the exponential series to include only the first order terms as follows:

$$
\frac{\varepsilon_{33}^{*}}{\varepsilon_{33}}=\left(1+\tan \delta^{\prime}+\tan \theta^{\prime}+\frac{C-C_{f}}{C_{f}}\right)^{2},
$$

we can find that low frequency behavior of ME coefficient is correlated with the loss dependence of dielectric constant. Thus if losses and relative change in capacitance are zero then the laminate will exhibit flat magnetoelectric response. The next question to answer is "what causes the increase in loss magnitudes at low frequencies?"

We think that increase in loss values at low frequencies and relative change in capacitance is related to the presence of charged defects induced by acceptor doping. Divalent and trivalent $\mathrm{Mn}$-ions coexist in manganese oxide above $\sim 870{ }^{\circ} \mathrm{C}$ and at $1 \mathrm{~atm}$ pressure. ${ }^{10}$ It is widely known that $\mathrm{Mn}$ ions incorporate onto B-site of the PZT ceramics and act as acceptors. ${ }^{11}$ Resulting oxygen vacancies pair with $\mathrm{Mn}^{3+}$ ions and form chains parallel to one of the crystallographic axes. These chain fragments interact with the lower valent species and result in polarization pinning. Since, the concentration of chain fragments and the associated cation pairing is very small, no long range ordering occurs. However, at low frequencies these charged chain fragments could accumulate in the vicinity of blocking electrodes and contribute to space charge polarization. This causes rapid increase in capaci$\operatorname{tance}, \tan \delta^{\prime}$ and $\tan \theta^{\prime}$ of the PMS. Flat response in ME voltage and loss factors of the PZN could be associated with the absence of defect-induced contribution. The experimental evidence for oxygen vacancy-induced space charge polarization at low frequencies is shown in Figs. 3(a) and 3(b). The PMS system exhibited rapid increase in remanent polarization $\left(\mathrm{P}_{\mathrm{r}}\right)$ and lossy hysteresis curve at low frequencies while the $\mathrm{PZN}$ system showed stable hysteresis and negligible 


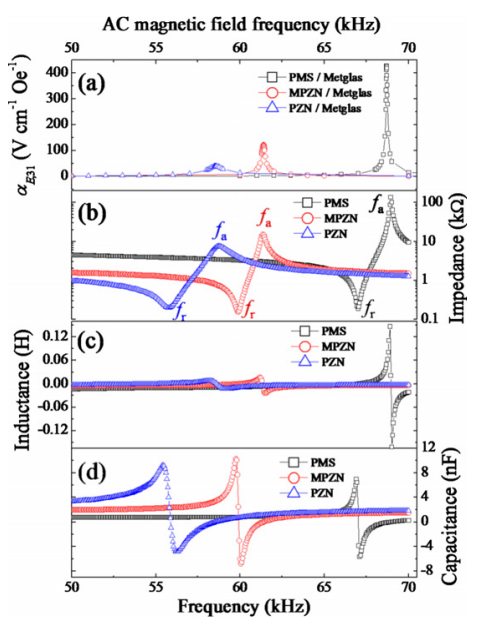

FIG. 4. (Color online) (a) Magnetoelectric voltage coefficient vs $\mathrm{H}_{\mathrm{ac}}$ frequency characteristics of PMS/Metglas, MPZN/Metglas, and PZN/Metglas laminates measured in the frequency range of $50-70 \mathrm{kHz}$. (b) impedance vs frequency, (c) inductance vs frequency and (d) capacitance vs frequency characteristics of PMS, MPZN, and PZN piezoelectric compositions measured in the frequency range of $50-70 \mathrm{kHz}$.

change in $\mathrm{P}_{\mathrm{r}}$. In order to further confirm the role of oxygen vacancies, we sintered the PMS sample in oxygen environment (flow rate of $\mathrm{O}_{2}=0.2 \mathrm{~L} \mathrm{~min}^{-1}$ ). Figure 3(c) shows the change in P-E behavior as a result of oxygen treatment clearly demonstrating the suppression of lossy behavior. The composition MPZN has much smaller Mn-doping (approximately five times), thus the effect of space charge polarization at low frequencies is also reduced.

Next, we investigated the ME behavior of laminate in high frequency resonance region. Mechanical loss, $\tan \varphi^{\prime}\left(=1 / \mathrm{Q}_{\mathrm{m}}\right)$, of piezoelectrics is an important factor for resonance mode operation of the ME composites. It has been reported that strong enhancement of ME voltage occurs at electromechanical resonance (EMR) frequency of piezoelectrics and the magnitude of enhancement is directly related to $\mathrm{Q}_{\mathrm{m}}$ value. ${ }^{1,12,13}$ PMS has a very high $\mathrm{Q}_{\mathrm{m}}$ and it exhibits giant $\alpha_{\mathrm{E}, 31}$ of $428 \mathrm{~V} \mathrm{~cm}^{-1} \mathrm{Oe}^{-1}(\sim 1400$ times higher than that at sub-resonance value) while PZN exhibits the lowest $\alpha_{\mathrm{E}, 31}$ of $39.6 \mathrm{~V} \mathrm{~cm}^{-1} \mathrm{Oe}^{-1}(\sim 188$ times higher than sub-resonance value). The other interesting observation was that maximum enhancement in $\alpha_{\mathrm{E}, 31}$ occurs at antiresonance frequency $\left(f_{\mathrm{a}}\right)$ of piezoelectrics (in fact, very far from the EMR) as shown in Figs. 4(a) and 4(b). Recent studies by Petrov and Bichurin also predicts similar observation. ${ }^{14}$ The magnitude of $Q_{a}$ for these three compositions was measured to be, $\mathrm{Q}_{\mathrm{a}, \mathrm{PMS}}=2318$, $\mathrm{Q}_{\mathrm{a}, \mathrm{MPZN}}=910$, and $\mathrm{Q}_{\mathrm{a}, \mathrm{PZN}}=109$, which is only slightly higher than $\mathrm{Q}_{\mathrm{r}}$ as shown in Table I. In Table II, we list the calculated

TABLE II. List of extensive loss factors.

\begin{tabular}{lccc}
\hline \hline & $\tan \delta$ & $\tan \phi$ & $\tan \theta$ \\
\hline PMS & 0.0124 & 0.00046 & 0.0066 \\
MPZN & 0.0126 & 0.00099 & 0.0038 \\
PZN & 0.0103 & 0.00848 & -0.00077 \\
\hline \hline
\end{tabular}

magnitude of extensive loss factors. ${ }^{6,7}$ It can be seen from this table that there is significant difference in the magnitude of extensive mechanical loss for the three compositions, with PMS exhibiting the lowest magnitude. Thus, high $\mathrm{Q}_{\mathrm{a}}$ and low extensive mechanical loss can explain the large ME coefficient of PMS but it does not provide explanation for peak occurring at $\mathrm{f}_{\mathrm{a}}$. We attempt to explain this finding by considering the equivalent circuit model for piezoelectric resonator.

Figures 4(c) and 4(d) show that the trend in increase of ME voltage coefficient near the $f_{\mathrm{a}}$ follows the behavior of inductance and not that of capacitance. The piezoelectric material is considered to act as an inductor between $f_{\mathrm{r}}$ and $f_{\mathrm{a}}$. Furthermore, near $f_{\mathrm{a}}$, capacitance goes close to zero as shown in Fig. 4(d) and inductance exhibits the maximum. Uchino ${ }^{15}$ has described the resonance and antiresonance behavior in terms of strain distribution in the material. In the resonance state, large strain amplitudes and large capacitance changes are induced, and the current can easily flow into the device. On the other hand, at antiresonance, the strain induced in the device compensates completely, resulting in no capacitance change, and the current cannot flow easily into the sample. In this case, an external applied stress will result in charge generation across lower capacitance amplitude, thereby generating higher voltage.

In summary, this study shows that the dynamic ME coefficient of piezoelectric/magnetostrictive laminates is affected by the loss factors. PMS/Metglas laminates exhibited high $\alpha_{\mathrm{E}, 31}$ mainly due to its high $g_{31}$, low $\tan \varphi^{\prime}$ and low $S_{11}^{E}$ but showed significant degradation at the low frequencies due to high $\tan \delta^{\prime}$ and $\tan \theta^{\prime}$. In EMR range, the magnitude of $\mathrm{ME}$ voltage coefficient corresponds with $1 / \tan \varphi$, with the peak occurring at $\mathrm{f}_{\mathrm{a}}$.

The authors gratefully acknowledge the financial support from DARPA Heterostructural Uncooled Magnetic Sensors (HUMS) program (Grant No. FA8650-09-1-7945).

${ }^{1}$ C.-W. Nan, M. I. Bichurin, S. Dong, D. Viehland, and G. Srinivasan, J. Appl. Phys. 103, 031101 (2008).

${ }^{2}$ G. Srinivasan, Annu. Rev. Mater. Res. 40, 153 (2010).

${ }^{3}$ M. Fiebig, J. Phys. D: Appl. Phys. 38, R123 (2005).

${ }^{4}$ M. I. Bichurin, R. V. Petrov, and Y. V. Kiliba, Ferroelectrics 204, 311 (1997)

${ }^{5}$ Y. K. Fetisov and G. Srinivasan, Appl. Phys. Lett. 88, 143503 (2006).

${ }^{6} \mathrm{~K}$. Uchino and S. Hirose, IEEE Trans. Ultrason. Ferroelectr. Freq. Control 48, 307 (2001)

${ }^{7}$ Y. Zhuang, S. O. Ural, A. Rajapurkar, S. Tuncdemir, A. Amin, and K. Uchino, Jpn. J. Appl. Phys. 48, 041401 (2009).

${ }^{8}$ R. Islam and S. Priya, J. Am. Ceram. Soc. 89, 3147 (2006)

${ }^{9}$ S. Priya, J. Ryu, K. Uchino, C. Ahn, and S. Nahm, Appl. Phys. Lett. 83, 5020 (2003).

${ }^{10}$ F. Shenouda and S. Aziz, J. Appl. Chem. 17, 258 (1967).

${ }^{11}$ A. J. Moulson and J. M. Herbert, Electroceramics (Chapman and Hall, New York, 1990).

${ }^{12}$ M. I. Bichurin, D. A. Filippov, and V. M. Petrov, Phys. Rev. B 68, 132408 (2003).

${ }^{13}$ S. X. Dong, J. R. Cheng, J. F. Li, and D. Viehland, Appl. Phys. Lett. 83, 4812 (2003)

${ }^{14}$ M. I. Bichurin and V. M. Petrov, Low Temp. Phys. 36, 544 (2010).

${ }^{15}$ K. Uchino, Ferroelectric Devices (CRC/Dekker, New York, 2000). 